\title{
Prevalência de Hipovitaminose de Associações com Parâmetros Metabólicos em Trabalhadores
}

\author{
Laine de Carvalho Guerra Pessoa Mamede, ${ }^{1}$ \\ Rafaela Lira Formiga Cavalcanti de Lima, ${ }^{2}$ Alexandre Sérgio Silva, ${ }^{1}$ \\ Rubens Batista Benedito, ${ }^{1}$ Lydiane de Lima Tavares Toscano, ${ }^{1}$ \\ Nadjeanny Ingrid Galdino Gomes, ${ }^{1}$ Elisama Araújo de Sena, ${ }^{1}$ \\ Maria da Conceição Rodrigues Gonçalves ${ }^{1}$
}

\section{RESUMO}

A hipovitaminose $D$ é considerada uma epidemia mundial com implicações que podem afetar a saúde dos trabalhadores. Trata-se de um estudo transversal, realizado com 91 trabalhadores de Unidades de Alimentação e Nutrição, a fim de avaliar a prevalência da hipovitaminose D e associações com parâmetros metabólicos. Foram estudados o perfil sociodemográfico e ocupacional, exposição solar, antropometria e pressão arterial. Foram realizados os exames: 25-hidroxivitamina D [25(OH)D] e demais parâmetros metabólicos. Verificou-se uma prevalência de $61,54 \%$ de deficiência/insuficiência dos níveis séricos de $25(\mathrm{OH}) \mathrm{D}$. Não foram observadas associações entre as características sociodemográficas e ocupacionais e o status de vitamina D, pela Regressão de Poisson. Verificou-se uma prevalência aumentada em portadores de diabetes e hipertrigliceridemia. Indivíduos com maior Capacidade Antioxidante Total (CAT) apresentaram uma redução na prevalência de hipovitaminose D. Em conclusão, constatou-se uma alta prevalência de hipovitaminose $D$ entre os trabalhadores, relacionada aos níveis aumentados de glicemia e triglicerídeos, e a CAT como influenciável para redução dessa deficiência/insuficiência. Enfatiza-se a necessidade de um monitoramento dos parâmetros laboratoriais em vigilância à saúde do trabalhador

Palavras-chave: Deficiência de vitamina D; estresse oxidativo; Diabetes Mellitus Tipo 2; saúde do trabalhador.

PREVALENCE OF HYPOVITAMINOSIS D AND ASSOCIATIONS WITH METABOLIC PARAMETERS IN WORKERS

\section{ABSTRACT}

Hypovitaminosis D is considered a worldwide epidemic with implications that may affect the health of workers. This is a cross-sectional study conducted with 91 workers from Food and Nutrition Units in order to assess the prevalence of hypovitaminosis D and the associations with metabolic parameters. Were evaluated regarding their sociodemographic and occupational profile, exposure to sunlight, anthropometric data and blood pressure. The following exams were performed: 25 -hydroxyvitamin $\mathrm{D}[25(\mathrm{OH}) \mathrm{D}]$ and other metabolic parameters. There was a prevalence of $61.54 \%$ of the deficiency/insufficiency of $25(\mathrm{OH}) \mathrm{D}$ serum levels. No associations were confirmed between the vitamin D status and the sociodemographic and occupational characteristics through the Poisson regression. There was an increased prevalence in patients with diabetes and hypertriglyceridemia. Individuals with high Total Antioxidant Capacity (TAC) values showed a reduction in the prevalence of hypovitaminosis D. In conclusion, a high prevalence of hypovitaminosis D was found among the workers, related to increased blood glucose and triglyceride levels with TAC proving susceptible to reduce this deficiency/insufficiency. The need to monitor laboratory parameters in the health surveillance of workers is stressed.

Keywords: Vitamin D deficiency; oxidative stress; Type 2 Diabetes Mellitus; occupational health.

RECEBIDO EM: 27/10/2020

MODIFICAÇÕES SOLICITADAS EM: 12/3/2021

ACEITO EM: 22/6/2021

\footnotetext{
${ }^{1}$ Universidade Federal da Paraíba

${ }^{2}$ Autora correspondente. Universidade Federal da Paraíba - Centro de Ciências da Saúde, Campus I, Departamento de Nutrição. Cidade Universitária - Castelo Branco. João Pessoa/PB, Brasil. CEP 58051-900. http:// lattes.cnpq.br/7251030533021754. https://orcid.org/0000-0003-1272-0067. rafaelanutri@gmail.com
} 


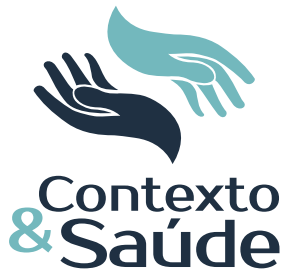

\section{INTRODUÇÃO}

A hipovitaminose $D$ é altamente prevalente em todo o mundo, sendo considerada um problema de saúde pública relacionado ao desenvolvimento de diversas enfermidades crônicas, como obesidade, hipertensão, câncer, Diabetes Tipo 2, síndrome metabólica, doenças autoimunes, entre outras. ${ }^{1-4}$.

Alguns estudos epidemiológicos têm constatado que, independentemente da idade, etnia e região geográfica, uma parcela significativa da população mundial apresenta baixos níveis séricos de 25-hidroxivitamina $D[25(\mathrm{OH}) \mathrm{D}]$. Em alguns países a prevalência da hipovitaminose $D$ atinge percentuais superiores a 50\%, como registrado no Brasil, Finlândia e Dinamarca. ${ }^{5,6}$

Em geral, os baixos níveis de vitamina D (VD) estão associados a vários fatores de riscos, como exposição solar inadequada, fototipo de pele, cobertura extensa de roupas, evitar a exposição ao sol com o uso de protetor sola, estilo de vida indoor (meio ambiente interno com privação de sol), poluição do ar, síndrome de má absorção, baixa ingestão de VD, entre outros. ${ }^{6-8}$

Nos Estados Unidos 42\% da população adulta se expõe ao risco de deficiência ou insuficiência de VD. As elevadas taxas de hipovitaminose D provavelmente estão relacionadas à crescente prevalência de obesidade e mudanças do estilo de vida. As atividades laborais que eram predominantemente externas passaram a ser realizadas em ambientes interiores com privação do sol, e adjacente à crescente preocupação com a redução do risco de câncer de pele, levando a uma menor exposição solar, que é o principal meio de síntese da VD9.

Em pesquisa realizada com uma população do Catar, apesar das extensas horas de luz solar, observou-se uma alta prevalência de deficiência de Vitamina $D$ e a sua associação ao diagnóstico de síndrome metabólica. Evidenciou-se também reduzidos níveis séricos $(<20 \mathrm{ng} / \mathrm{mL})$ de $25(\mathrm{OH}) \mathrm{D}$ nos participantes de um estudo desenvolvido no Kwait (98\%) e nos Emirados (83\%), ${ }^{1}$ assim como na China; em comunidades urbanas e suburbanas de Shanghai a situação é consideravelmente preocupante, em que a prevalência de deficiência de VD $(<20 \mathrm{ng} / \mathrm{mL})$ observada foi de $35,4 \%$ dos homens e $50,5 \%$ das mulheres, com níveis médios de $25(\mathrm{OH}) \mathrm{D}$ de $22,73 \mathrm{ng} / \mathrm{mL}$ e $19,99 \mathrm{ng} / \mathrm{mL}$, respectivamente. ${ }^{10}$

No Brasil, estudo desenvolvido com indivíduos saudáveis constatou uma prevalência de $77,4 \%$ de hipovitaminose $D$ após o inverno, apresentando os níveis de $25(\mathrm{OH}) \mathrm{D}$ dependentes da idade e fototipo de pele. Após o verão observou-se uma diminuição da prevalência de hipovitaminose $D$, e essa melhora foi dependente da idade. ${ }^{11}$

A hipovitaminose $D$ atinge populações em diferentes faixas etárias em todo mundo, no entanto estudos sobre o status da VD de acordo com a atividade laboral ainda são escassos. ${ }^{12}$

Pesquisa desenvolvida com trabalhadores na área de saúde demonstrou alta prevalência de deficiência de VD (69,3\%), devido à permanência em ambientes internos, sem exposição solar, pelas suas atividades ocupacionais em ambientes hospitalares, o que justifica o desenvolvimento de estudo sobre os níveis de 25(OH)D nesses trabalhadores. ${ }^{13}$ 
Diante do exposto, sabe-se que a deficiência ou insuficiência de VD é um problema de saúde em todo o mundo, destacando-se como principais causas a menor exposição ao sol e transformações no estilo de vida. Nesse sentido, ressalta-se a preocupação com a saúde dos trabalhadores ante os fatores de riscos, que estão expostos, para a hipovitaminose $\mathrm{D}$ no desenvolvimento das suas atividades ocupacionais. O estudo teve por objetivo avaliar a prevalência da hipovitaminose $D$ e as associações com os parâmetros metabólicos em trabalhadores de Unidades de Alimentação e Nutrição.

\section{MÉTODO}

Trata-se de um estudo transversal, realizado com trabalhadores de Unidades de Alimentação e Nutrição, da Universidade Federal da Paraíba, Campus I, aprovado por Comitê de Ética em Pesquisa, atendendo à Resolução no 466/2012 do Conselho Nacional de Saúde. ${ }^{14}$ Todos os participantes assinaram o Termo de Consentimento Livre e Esclarecido.

$\mathrm{O}$ estudo foi realizado com trabalhadores de ambos os sexos. A amostra foi coletada por conveniência, inicialmente foram incluídos na amostra 91 trabalhadores. Os critérios de elegibilidade foram idade entre 18 anos e 59 anos; não fazer suplementação com VD; não fazer uso de anticonvulsivantes ou medicamentos para tratamento do Vírus da Imunodeficiência Humana (HIV) ou da Síndrome da Imunodeficiência Adquirida (Aids); sem diagnóstico de Diabetes Mellitus tipo 1, síndrome nefrótica, insuficiência renal aguda ou crônica, hepatopatias, hipotireoidismo, hipertireoidismo, história de Acidente Vascular Cerebral (AVC) ou Infarto Agudo do Miocárdio (IAM) nos últimos 6 meses; não elitistas e/ ou tabagistas crônicos.

Para traçar o perfil sociodemográfico e ocupacional da amostra estudada foram aplicados questionários estruturados, a fim de conhecer: características pessoais (sexo, faixa etária e escolaridade) e ocupacionais (tempo de serviço na instituição/empresa e renda per capita familiar.

Os fototipos de pele foram classificados de acordo com a Escala de Fitzpatrick, com variação de um a seis tipos, a partir da descrição da pele; se queima com facilidade, pouco, raramente ou nunca; capacidade de se bronzear; sensibilidade ao sol, variando do pouco sensível ao muito sensível ${ }^{15}$. Cada participante respondeu quanto ao seu fototipo de pele e, após isso, foram considerados: não negros (fototipos de I a IV) e negros (fototipos de V e VI). Quanto à exposição solar foi definida pelo tempo médio de exposição ao dia, não considerando as variações sazonais.

Os dados antropométricos foram coletados em duplicata e pelo mesmo avaliador, de acordo com as recomendações e classificações da World Health Organization. ${ }^{16} \mathrm{~A}$ avaliação do estado nutricional foi determinada pelo Índice de Massa Corporal (IMC). ${ }^{16}$

Para analisar a obesidade abdominal a medição da Circunferência da Cintura (CC) foi realizada no ponto médio entre a face externa da última costela e a crista ilíaca, com leitura no momento da expiração e classificada em dois níveis, considerando riscos de complicações metabólicas associadas à obesidade: para 
as mulheres, nível 1 ( $\geq 80 \mathrm{~cm}$ e $<88 \mathrm{~cm})$ - risco elevado, e o nível $2(\geq 88 \mathrm{~cm})-$ muito elevado; para os homens, nível 1 ( $\geq 94 \mathrm{~cm}$ e $<102 \mathrm{~cm}$ ) - risco elevado, e o nível 2 ( $\geq 102 \mathrm{~cm})$ - muito elevado. ${ }^{16}$

Procedeu-se à verificação da Pressão Arterial (PA), de acordo com a 7ạ Diretriz Brasileira de Hipertensão Arterial. ${ }^{17} \mathrm{~A}$ aferição foi realizada em triplicata com intervalo de 1 minuto entre as aferições, enquanto o paciente estava sentado em repouso por pelo menos 5 minutos, sem as pernas estarem cruzadas e com o braço apoiado no nível do coração. ${ }^{18}$

Para a coleta do material bioquímico analisado os participantes foram orientados a realizar jejum de 12 horas para a realização da coleta de sangue. As análises bioquímicas realizadas foram das funções renal e hepática, hemograma completo, perfis glicêmico e lipídico.

As concentrações séricas de 25(OH)D foram mensuradas por imunoensaio quimioluminescente. A classificação dos níveis de 25(OH)D foi realizada com base nos valores de referência estabelecidos pela Endocrine Society, que considera deficiente nível sérico de $25(\mathrm{OH}) \mathrm{D}$ menor ou igual a $20 \mathrm{ng} / \mathrm{mL}$, insuficiente entre $21-29 \mathrm{ng} / \mathrm{mL}$ e suficiente entre $30-100 \mathrm{ng} / \mathrm{mL}^{19}$

Os níveis séricos de Paratormônio (PTH) foram avaliados por ensaio imunométrico quimioluminescente, tendo como valores de referência $10-65 \mathrm{pg} / \mathrm{mL}$. As concentrações séricas de Cálcio (Ca) foram mensuradas por técnica colorimétrica com valores de referência entre 8,4 e $10,2 \mathrm{mg} /{ }^{21} \mathrm{dL} .{ }^{20}$

Os marcadores inflamatórios foram analisados através da Proteína C Reativa (PCR) e da Alfa-1 Glicoproteína Ácida (AGP) pelo método imunoturbidimétrico, com valores de referência de 0 a 5,0 mg/L e de 0 a $150 \mathrm{mg} / \mathrm{L}$, respectivamente.

O estresse oxidativo foi medido pela análise da Capacidade Antioxidante Total (CAT), pelo método do sequestro do radical livre estável, 2,2-difenil-1-picrilhidrazil (DPPH) e, da peroxidação lipídica, baseada nas espécies reativas ao ácido tiobarbitúrico (TBARS), por ensaio espectrofotométrico, em que os resultados foram calculados de acordo com a curva-padrão feita com Malondialdeído (MDA). ${ }^{21,22}$ Por não apresentar Valor de Referência (VR) consolidado pela literatura, a variável contínua CAT foi dicotomizada considerando a média $(24,80 \%)$ devido à distribuição normal. Com a mesma justificativa o MDA foi categorizado, porém, por não apresentar distribuição normal, a referência foi a mediana $(2,90$ $\mu \mathrm{mol} / \mathrm{L})$.

\section{Análises estatísticas}

Os dados foram analisados no Stata Statistical Software, versão 14. Os resultados das variáveis sociodemográficas e ocupacional, exposição e proteção solar e de estado nutricional foram descritos de suas respectivas frequências. Para avaliar as associações entre essas variáveis e o status de 25(OH)D foi utilizado o teste de independência Qui-quadrado.

As variáveis referentes aos parâmetros bioquímicos e marcadores inflamatórios foram descritas por meio da utilização de medidas de tendência central e dispersão, o Teste-t para amostras independentes foi utilizado para comparar as médias desses marcadores para os trabalhadores classificados com status de VD

Editora Unijuí - Revista Contexto \& Saúde - ISSN 2176-7114 - v. 21, n. 44, out./dez. 2021 
deficiente/insuficiente com daqueles suficientes. O modelo de regressão linear múltipla de Poisson com variância robusta foi utilizado para estimar a Razão de Prevalência (RP) bruta, modelo univariado e RP ajustada entre todas as variáveis independentes e o status de 25(OH)D. Em todo o estudo foram considerados significativos os testes cujo $\mathrm{p}$-valor foi $<0,05$.

\section{RESULTADOS}

Participaram do presente estudo 91 trabalhadores (31 homens e 60 muIheres) de Unidades de Alimentação e Nutrição, com idade média de aproximadamente $41 \pm 10,3$ anos e não houve diferenças entre as médias de idade entre os grupos formados conforme status de VD. Em relação ao tempo de serviço na unidade, 47 trabalhadores tinham igual ou menor que 24 meses e 44 apresentavam tempo na empresa maior que 24 meses. A renda mediana da amostra foi de $\mathrm{R} \$ 655,88$, aproximadamente $70 \%$ do valor do salário-mínimo vigente na época da coleta de dados.

Para os níveis de 25(OH)D, foi observada uma prevalência de $61,54 \%$ $(n=56)$ de hipovitaminose $D$, em que 47 trabalhadores com deficiência/insuficiência de VD foram diagnosticados com sobrepeso ou obesidade. Das variáveis relacionadas aos perfis sociodemográfico e ocupacional, fototipo de pele e tempo de exposição e proteção solar, pressão arterial sistólica e diastólica, estado nutricional e CC, não foram constatadas diferenças significativas em relação ao status de VD, com exceção da "Se expõe ao sol na ida ao trabalho", que apresentou associação com o status da 25(OH)D $(p<0,025)$. As características gerais dos participantes do estudo e a associação com status de 25(OH)D estão apresentadas na Tabela 1.

Tabela 1 - Descrição das frequências das variáveis sociodemográfica e ocupacional, fototipo de pele, exposição e proteção solar, pressão arterial sistólica e diastólica, estado nutricional e circunferência da cintura para amostra total e associação com status de 25-hidroxivitamina D em trabalhadores, João Pessoa - PB

\begin{tabular}{|c|c|c|c|c|c|c|c|}
\hline \multirow[b]{3}{*}{ Variáveis } & \multicolumn{7}{|c|}{ 25-hidroxivitamina D } \\
\hline & \multicolumn{2}{|c|}{ Deficiência } & \multicolumn{2}{|c|}{ Suficiente } & \multicolumn{2}{|c|}{ Total } & \multirow[b]{2}{*}{ p-valor } \\
\hline & $\mathrm{n}$ & $\%$ & $\mathrm{~N}$ & $\%$ & $\mathrm{n}$ & $\%$ & \\
\hline \multicolumn{8}{|l|}{ Escolaridade } \\
\hline Até 1으 Grau & 20 & 55,6 & 16 & 44,4 & 36 & 100 & \\
\hline Até 20 Grau ou mais & 36 & 65,5 & 19 & 34,5 & 55 & 100 & $0,34 *$ \\
\hline \multicolumn{8}{|l|}{ Tempo de Serviço } \\
\hline$\leq 24$ meses & 29 & 61,7 & 18 & 38,3 & 47 & 100 & \\
\hline$>24$ meses & 27 & 61,4 & 17 & 38,6 & 44 & 100 & $0,97 *$ \\
\hline \multicolumn{8}{|l|}{ Fototipo } \\
\hline Não negros & 45 & 60,8 & 29 & 39,2 & 74 & 100 & \\
\hline Negros & 11 & 64,7 & 6 & 35,3 & 17 & 100 & $0,77^{*}$ \\
\hline \multicolumn{8}{|c|}{$\begin{array}{l}\text { Tempo de exposição ao } \\
\text { sol }\end{array}$} \\
\hline$\leq 15$ minutos & 32 & 71,1 & 13 & 28,9 & 45 & 100 & \\
\hline$>15$ minutos & 24 & 52,2 & 22 & 47,8 & 46 & 100 & $0,06^{*}$ \\
\hline
\end{tabular}




\begin{tabular}{|c|c|c|c|c|c|c|c|}
\hline $\begin{array}{l}\text { Uso de protetor ao dia } \\
1 \text { vez ao dia } \\
\text { Mais de uma vez ao dia }\end{array}$ & $\begin{array}{c}23 \\
9\end{array}$ & $\begin{array}{l}74,2 \\
90,0\end{array}$ & $\begin{array}{l}8 \\
1\end{array}$ & $\begin{array}{l}25,8 \\
10,0\end{array}$ & $\begin{array}{l}31 \\
10\end{array}$ & $\begin{array}{l}100 \\
100\end{array}$ & $0,29^{\#}$ \\
\hline \multicolumn{8}{|l|}{$\begin{array}{l}\text { Forma de uso do } \\
\text { protetor solar }\end{array}$} \\
\hline Diariamente & 12 & 70,6 & 5 & 29,4 & 17 & 100 & \\
\hline Quando se expõe ao sol & 15 & 68,2 & 7 & 31,8 & 22 & 100 & $0,28^{\#}$ \\
\hline \multicolumn{8}{|l|}{ PAS } \\
\hline Normal & 30 & 68,2 & 14 & 31,8 & 44 & 100 & \\
\hline Pré-hipertensão arterial & 17 & 48,6 & 18 & 51,4 & 35 & 100 & \\
\hline Hipertensão arterial & 9 & 75,0 & 3 & 25,0 & 12 & 100 & $0,12^{\#}$ \\
\hline \multicolumn{8}{|l|}{ PAD } \\
\hline Normal & 31 & 66,0 & 16 & 34 & 47,0 & 100 & \\
\hline Pré-hipertensão arterial & 15 & 60,0 & 10 & 40 & 25,0 & 100 & \\
\hline Hipertensão arterial & 9 & 56,3 & 7 & 43,8 & 16,0 & 100 & $0,75^{*}$ \\
\hline \multicolumn{8}{|l|}{$\begin{array}{l}\text { Se expõe ao sol na ida ao } \\
\text { trabalho }\end{array}$} \\
\hline Não & 38 & 55,1 & 31 & 44,9 & 69 & 100 & \\
\hline Sim & 18 & 81,8 & 4 & 18,2 & 22 & 100 & $0,03^{\#}$ \\
\hline \multicolumn{8}{|l|}{ CC } \\
\hline Adequado & 13 & 61,9 & 8 & 38,1 & 21 & 100 & \\
\hline Risco & 43 & 61,4 & 27 & 38,6 & 70 & 100 & $0,97^{*}$ \\
\hline \multicolumn{8}{|l|}{ Estado nutricional } \\
\hline Desnutrição & 2 & 66,7 & 1 & 33,3 & 3 & 100 & \\
\hline Eutrofia & 7 & 58,3 & 5 & 41,7 & 12 & 100 & \\
\hline Sobrepeso/obesidade & 47 & 61,8 & 29 & 38,2 & 76 & 100 & $0,96^{\#}$ \\
\hline Total & 56 & 61,5 & 35 & 38,5 & 91 & 100 & \\
\hline
\end{tabular}

PAS: Pressão Arterial Sistólica; PAD: Pressão Arterial Diastólica; CC: Circunferência da Cintura.

*Teste Qui-quadrado; \# Exato de Fisher para os momentos em que a frequência esperada foi menor ou igual a 5. Fonte: Dados da pesquisa, 2019.

Na Tabela 2 estão descritos as médias e os desvios-padrão das variáveis relativas aos parâmetros bioquímicos, marcadores inflamatórios e de estresse oxidativo. Pode-se observar que para as variáveis bioquímicas relacionadas ao hemograma, glicemia de jejum, perfil lipídico, funções renal e hepática e para os parâmetros inflamatórios (PCR e AGP), não existem diferenças entre as médias com o status de 25(OH)D. Em relação aos marcadores de estresse oxidativo, não houve diferença entre as concentrações médias de MDA, porém, para a CAT observa-se que houve diferença $(p=0,004)$ em relação ao status de VD, indivíduos com suficiência de VD apresentam maior capacidade antioxidante do que aqueles com deficiência/insuficiência.

Tabela 2 - Comparação das médias de parâmetros bioquímicos, marcadores inflamatórios e de estresse oxidativo entre os níveis de 25-hidroxivitamina D em trabalhadores, João Pessoa - PB

\begin{tabular}{lcccc}
\hline \multicolumn{1}{c}{ 25-hidroxivitamina D } & $\mathbf{n}$ & Média & Desvio padrão & p-valor* \\
\hline Hemácias & & & & \\
Deficiente/Insuficiente & 56 & 4,751 & 0,485 & \\
Suficiente & 35 & 4,711 & 0,489 & 0,71 \\
\hline Hemoglobina & & & & \\
Deficiente/Insuficiente & 56 & 13,557 & 1,262 & \\
Suficiente & 35 & 13,588 & 1,916 & 0,93 \\
\hline
\end{tabular}




\begin{tabular}{|c|c|c|c|c|}
\hline $\begin{array}{l}\text { Leucócitos } \\
\text { Deficiente/Insuficiente } \\
\text { Suficiente } \\
\end{array}$ & $\begin{array}{l}56 \\
35 \\
\end{array}$ & $\begin{array}{l}7443,21 \\
7522,00 \\
\end{array}$ & $\begin{array}{l}2152,99 \\
1895,61 \\
\end{array}$ & 0,86 \\
\hline $\begin{array}{l}\text { Linfócitos } \\
\text { Deficiente/Insuficiente } \\
\text { Suficiente }\end{array}$ & $\begin{array}{l}56 \\
35\end{array}$ & $\begin{array}{l}2409,12 \\
2351,11\end{array}$ & $\begin{array}{l}665,25 \\
578,49\end{array}$ & 0,66 \\
\hline $\begin{array}{l}\text { Plaquetas } \\
\text { Deficiente/Insuficiente } \\
\text { Suficiente }\end{array}$ & $\begin{array}{l}56 \\
35 \\
\end{array}$ & $\begin{array}{l}256178,57 \\
249657,14\end{array}$ & $\begin{array}{l}68842,93 \\
73046,29 \\
\end{array}$ & 0,67 \\
\hline $\begin{array}{l}\text { Glicemia de Jejum } \\
\text { Deficiente/Insuficiente } \\
\text { Suficiente }\end{array}$ & $\begin{array}{l}56 \\
35 \\
\end{array}$ & $\begin{array}{c}100,428 \\
93,114\end{array}$ & $\begin{array}{l}33,787 \\
13,150\end{array}$ & 0,15 \\
\hline $\begin{array}{l}\text { Ureia } \\
\text { Deficiente/Insuficiente } \\
\text { Suficiente }\end{array}$ & $\begin{array}{l}56 \\
35 \\
\end{array}$ & $\begin{array}{l}30,535 \\
31,485\end{array}$ & $\begin{array}{l}6,9882 \\
9,6050\end{array}$ & 0,59 \\
\hline $\begin{array}{l}\text { Creatinina sérica Deficiente/ } \\
\text { Insuficiente } \\
\text { Suficiente }\end{array}$ & $\begin{array}{l}56 \\
35 \\
\end{array}$ & $\begin{array}{l}0,8129 \\
0,8691\end{array}$ & $\begin{array}{l}0,1510 \\
0,1482\end{array}$ & 0,08 \\
\hline $\begin{array}{l}\text { AUS } \\
\text { Deficiente/Insuficiente } \\
\text { Suficiente }\end{array}$ & $\begin{array}{l}56 \\
35 \\
\end{array}$ & $\begin{array}{l}4,5518 \\
5,0857 \\
\end{array}$ & $\begin{array}{l}2,3277 \\
2,6676 \\
\end{array}$ & 0,32 \\
\hline $\begin{array}{l}\text { Colesterol total Deficiente/ } \\
\text { Insuficiente } \\
\text { Suficiente }\end{array}$ & $\begin{array}{l}56 \\
35 \\
\end{array}$ & $\begin{array}{l}198,410 \\
197,228\end{array}$ & $\begin{array}{l}44,0519 \\
39,7611\end{array}$ & 0,90 \\
\hline $\begin{array}{l}\text { HDL } \\
\text { Deficiente/Insuficiente } \\
\text { Suficiente }\end{array}$ & $\begin{array}{l}56 \\
35 \\
\end{array}$ & $\begin{array}{l}46,957 \\
45,857 \\
\end{array}$ & $\begin{array}{l}9,4274 \\
8,1864 \\
\end{array}$ & 0,61 \\
\hline $\begin{array}{l}\text { LDL** } \\
\text { Deficiente/Insuficiente } \\
\text { Suficiente }\end{array}$ & $\begin{array}{l}55 \\
34\end{array}$ & $\begin{array}{l}124,161 \\
120,208\end{array}$ & $\begin{array}{l}35,128 \\
35,258\end{array}$ & 0,61 \\
\hline
\end{tabular}

25(OH)D: 25hidroxivitamina D; AUS: Ácido Úrico Sérico; HDL: Lipoproteína de Alta Densidade; LDL: Lipoproteína de Baixa Densidade.

*Teste T para amostras independentes.

**Ausência de dois resultados ( $n=89$ ), devido: 1) O cálculo não pode ser realizado com triglicerídeo maior ou igual a $400 \mathrm{mg} / \mathrm{dL}$; 2) Não existem valores definidos de VLDL, comprovadamente associados a um menor risco cardiovascular.

Fonte: Dados da pesquisa.

Tabela 3 - Comparação das médias de parâmetros bioquímicos, marcadores inflamatórios e de estresse oxidativo entre os níveis de 25-hidroxivitamina D em trabalhadores, João Pessoa - PB (continuação)

\begin{tabular}{|c|c|c|c|c|}
\hline 25-hidroxivitamina D & $\mathbf{n}$ & Média & Desvio padrão & p-valor* \\
\hline $\begin{array}{l}\text { VLDL** } \\
\text { Deficiente/Insuficiente } \\
\text { Suficiente }\end{array}$ & $\begin{array}{l}55 \\
34 \\
\end{array}$ & $\begin{array}{l}26,589 \\
30,217\end{array}$ & $\begin{array}{l}14,652 \\
16,447 \\
\end{array}$ & 0,28 \\
\hline $\begin{array}{l}\text { Triglicerídeos Deficiente } \\
\text { Insuficiente } \\
\text { Suficiente }\end{array}$ & $\begin{array}{l}56 \\
35\end{array}$ & $\begin{array}{l}143,910 \\
158,800\end{array}$ & $\begin{array}{c}100,246 \\
92,981\end{array}$ & 0,47 \\
\hline $\begin{array}{l}\text { AST } \\
\text { Deficiente/Insuficiente } \\
\text { Suficiente }\end{array}$ & $\begin{array}{l}56 \\
35 \\
\end{array}$ & $\begin{array}{l}21,646 \\
20,625 \\
\end{array}$ & $\begin{array}{l}11,867 \\
7,0855 \\
\end{array}$ & 0,65 \\
\hline $\begin{array}{l}\text { ALT } \\
\text { Deficiente/Insuficiente } \\
\text { Suficiente }\end{array}$ & $\begin{array}{l}56 \\
35\end{array}$ & $\begin{array}{l}26,464 \\
23,080\end{array}$ & $\begin{array}{l}20,427 \\
12,588\end{array}$ & 0,38 \\
\hline
\end{tabular}




\begin{tabular}{|c|c|c|c|c|}
\hline $\begin{array}{l}\text { Cálcio sérico total } \\
\text { Deficiente/Insuficiente } \\
\text { Suficiente }\end{array}$ & $\begin{array}{l}56 \\
35\end{array}$ & $\begin{array}{l}16,685 \\
13,660\end{array}$ & $\begin{array}{l}22,527 \\
20,025\end{array}$ & 0,52 \\
\hline $\begin{array}{l}\text { Paratormônio Deficiente/ } \\
\text { Insuficiente } \\
\text { Suficiente }\end{array}$ & $\begin{array}{l}56 \\
35\end{array}$ & $\begin{array}{l}42,757 \\
37,711\end{array}$ & $\begin{array}{l}13,260 \\
14,217\end{array}$ & 0,09 \\
\hline $\begin{array}{l}\text { Proteína C reativa Deficiente/ } \\
\text { Insuficiente } \\
\text { Suficiente }\end{array}$ & $\begin{array}{l}56 \\
35 \\
\end{array}$ & $\begin{array}{l}5,132 \\
5,577 \\
\end{array}$ & $\begin{array}{l}5,743 \\
7,805 \\
\end{array}$ & 0,76 \\
\hline $\begin{array}{l}\text { AGP } \\
\text { Deficiente/Insuficiente } \\
\text { Suficiente }\end{array}$ & $\begin{array}{l}56 \\
35 \\
\end{array}$ & $\begin{array}{l}88,705 \\
86,437\end{array}$ & $\begin{array}{l}20,937 \\
28,070\end{array}$ & 0,66 \\
\hline $\begin{array}{l}\text { CAT } \\
\text { Deficiente/Insuficiente } \\
\text { Suficiente }\end{array}$ & $\begin{array}{l}56 \\
35 \\
\end{array}$ & $\begin{array}{l}22,30 \\
28,80 \\
\end{array}$ & $\begin{array}{c}9,244 \\
11,659 \\
\end{array}$ & 0,00 \\
\hline $\begin{array}{l}\text { MDA } \\
\text { Deficiente/Insuficiente } \\
\text { Suficiente }\end{array}$ & $\begin{array}{l}56 \\
35 \\
\end{array}$ & $\begin{array}{l}3,093 \\
2,966 \\
\end{array}$ & $\begin{array}{l}0,9703 \\
0,6730 \\
\end{array}$ & 0,50 \\
\hline
\end{tabular}

25(OH)D: 25hidroxivitamina D; VLDL: Lipoproteína de Muito Baixa Densidade; AST: Aspartato Aminotransferase; ALT: Alanina Aminotransferase; AGP: Alfa Glicoproteína; CAT: Capacidade Antioxidante Total; MDA: Malondialdeído

*Teste T para amostras independentes.

**Ausência de dois resultados ( $n=89$ ), devido: 1) O cálculo não pode ser realizado com triglicerídeo maior ou igual a 400mg/dL; 2) Não existem valores definidos de VLDL, comprovadamente associados a um menor risco cardiovascular.

\section{Fonte: Dados da pesquisa.}

Com o objetivo de verificar associações entre as características dos trabaIhadores com o status de 25(OH)D, mesmo sem apresentar diferenças significantes na estatística univariada, um conjunto de variáveis foi incluído no Modelo de Regressão Múltipla de Poisson para ter os efeitos (razão de prevalência ajustada) estimados de forma independente para cada uma das variáveis (Tabela 4). 0 modelo apresentado reforça os achados da Tabela 1, no que diz respeito à ausência de associação entre as variáveis listadas com o status de VD. A exposição solar na ida ao trabalho apresenta associação no modelo univariado, porém esta não é confirmada no modelo ajustado.

Tabela 4. Razão de prevalência das variáveis sociodemográfica e ocupacional, fototipo de pele, exposição e proteção solar, pressão arterial sistólica e circunferência da cintura, associação com status de 25-hidroxivitamina D em trabalhadores, João Pessoa - PB

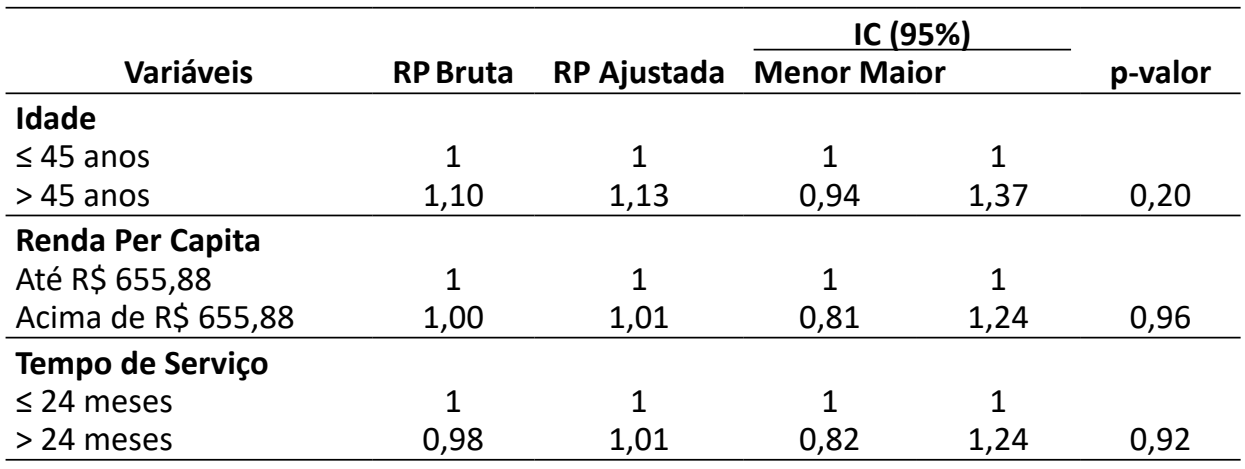




\begin{tabular}{lccccc}
\hline $\begin{array}{l}\text { Fototipos de pele } \\
\text { Fototipos: V e VI }\end{array}$ & 1 & 1 & 1 & 1 & \\
Fototipos: I, II, III e IV & 1,02 & 0,92 & 0,68 & 1,26 & 0,62 \\
\hline $\begin{array}{l}\text { Quantidade de protetor } \\
\text { Diariamente }\end{array}$ & 1 & 1 & 1 & 1 & \\
Quando se expõe ao sol & 0,98 & 1,01 & 0,79 & 1,27 & 0,97 \\
\hline $\begin{array}{l}\text { Se expõe ao sol na ida } \\
\text { ao trabalho }\end{array}$ & 1 & & & & \\
Não & $1,22^{*}$ & 1,14 & 0,90 & 1,43 & 0,27 \\
Sim & 1 & 1 & & & \\
\hline $\begin{array}{l}\text { PAS } \\
\text { Normal }\end{array}$ & 1,87 & 0,96 & 0,75 & 1,24 & 0,79 \\
$\begin{array}{l}\text { Pré-hipertensão arterial } \\
\text { Hipertensão arterial }\end{array}$ & 1,05 & 1,05 & 0,75 & 1,49 & 0,74 \\
\hline CC & 1 & & & & \\
Adequado & 1,00 & 1,02 & 0,79 & 1,31 & 0,89 \\
Risco & & & & & \\
\hline
\end{tabular}

PAS: Pressão Arterial Sistólica; CC: Circunferência da Cintura.

RP: Razão de Prevalência; ${ }^{*} p<0,05$ no modelo univariado.

Fonte: Dados da pesquisa.

Semelhantemente, as variáveis dos parâmetros bioquímicos, marcadores inflamatórios e de estresse oxidativo foram inseridos no modelo de Regressão de Poisson para calcular os efeitos, razão de prevalência bruta e ajustada entre as variáveis (Tabela 5). Indivíduos portadores de Diabetes Melito tipo 2 apresentam uma prevalência de hipovitaminose $D$ aumentada em $42 \%$. Em indivíduos com triglicerídeos muito altos ( $\geq 500 \mathrm{mg} / \mathrm{dL}$ ) a prevalência da deficiência/insuficiência é elevada em $46 \%$.

Tabela 5 - Razão de prevalência dos parâmetros bioquímicos, marcadores inflamatórios e de estresse oxidativo, associação com status de 25-hidroxivitamina D em trabalhadores, João Pessoa - PB

\begin{tabular}{|c|c|c|c|c|c|}
\hline \multirow{2}{*}{$\begin{array}{c}\text { Variáveis } \\
\text { Hemácias }\end{array}$} & RP Bruta & RP Ajustada & \multicolumn{2}{|c|}{$\frac{\text { IC (95\%) }}{\text { Inferior Superior }}$} & p-valor \\
\hline & & & & & \\
\hline Normal & 1 & 1 & 1 & 1 & \\
\hline Alterado & 0,85 & 0,85 & 0,67 & 1,08 & 0,17 \\
\hline \multicolumn{6}{|l|}{ Leucócitos } \\
\hline Normal & 1 & 1 & 1 & 1 & \\
\hline Alterado & 1,04 & 1,21 & 0,96 & 1,52 & 0,11 \\
\hline \multicolumn{6}{|l|}{ Plaquetas } \\
\hline Abaixo do VR & 1 & 1 & 1 & 1 & \\
\hline Normal & 0,96 & 1,11 & 0,70 & 1,77 & 0,63 \\
\hline Acima do VR & 0,89 & 0,94 & 0,54 & 1,63 & 0,84 \\
\hline \multicolumn{6}{|l|}{ Glicose } \\
\hline Normal & 1 & 1 & 1 & 1 & \\
\hline Glicose de jejum & 1,02 & 1,02 & 0,84 & 1,25 & 0,78 \\
\hline Diabetes tipo 2 & 1,42 & 1,42 & 1,14 & 1,78 & 0,00 \\
\hline \multicolumn{6}{|l|}{ Creatinina } \\
\hline Normal & 1 & 1 & 1 & 1 & \\
\hline Alterado & 1,04 & 1,16 & 0,83 & 1,60 & 0,38 \\
\hline
\end{tabular}




\begin{tabular}{|c|c|c|c|c|c|}
\hline \multicolumn{6}{|l|}{ Colesterol Total } \\
\hline Ótimo & 1 & 1 & 1 & 1 & \\
\hline Limítrofe & 1,12 & 1,13 & 0,96 & 1,32 & 0,13 \\
\hline Alto & 0,99 & 0,99 & 0,80 & 1,21 & 0,92 \\
\hline \multicolumn{6}{|l|}{ HDL } \\
\hline Baixo & 1 & 1 & 1 & 1 & \\
\hline Desejável & 0,99 & 0,96 & 0,67 & 1,14 & 0,73 \\
\hline \multicolumn{6}{|l|}{ Triglicerídeos } \\
\hline Desejável & 1 & 1 & 1 & 1 & \\
\hline Limítrofe & 0,95 & 0,92 & 0,77 & 1,11 & 0,42 \\
\hline Alto & 0,90 & 0,90 & 0,71 & 1,13 & 0,38 \\
\hline Muito alto & $1,34^{*}$ & 1,46 & 1,08 & 1,98 & 0,01 \\
\hline \multicolumn{6}{|l|}{ AST } \\
\hline Abaixo do VR & 1 & 1 & 1 & 1 & \\
\hline Normal & 1,20 & 1,04 & 0,60 & 1,81 & 0,88 \\
\hline Acima do VR & 1,33 & 1,24 & 0,66 & 2,35 & 0,50 \\
\hline \multicolumn{6}{|l|}{ Cálcio } \\
\hline Normal & 1 & 1 & 1 & 1 & \\
\hline Alterado & 1,00 & 1,06 & 0,80 & 1,41 & 0,67 \\
\hline \multicolumn{6}{|l|}{ Alfa1 glicoproteína } \\
\hline Normal & 1 & 1 & 1 & 1 & \\
\hline Alterado & 0,94 & 1,08 & 0,86 & 1,36 & 0,49 \\
\hline \multicolumn{6}{|l|}{ CAT } \\
\hline Abaixo da média & 1 & 1 & 1 & 1 & \\
\hline Acima da média & $0,84^{*}$ & 0,79 & 0,68 & 0,93 & 0,00 \\
\hline \multicolumn{6}{|l|}{ MDA } \\
\hline Abaixo da mediana & 1 & 1 & 1 & 1 & \\
\hline Acima da Mediana & 0,97 & 0,92 & 0,78 & 1,09 & 0,33 \\
\hline
\end{tabular}

VR: Valor de Referência.

RP: Razão de Prevalência; * $p<0,05$ no modelo univariado.

Fonte: Dados da pesquisa.

Com relação aos marcadores de estresse oxidativo, a capacidade antioxidante total apresenta-se como uma variável protetora para a deficiência de VD. Indivíduos com valores acima da média da variável para o grupo de trabalhadores apresentam uma prevalência reduzida em $21 \%$. Por outro lado, em relação ao Malondialdeído não foram observadas associações.

\section{DISCUSSÃO}

O presente estudo avaliou a prevalência de hipovitaminose $\mathrm{D}$ em trabalhadores de Unidades de Alimentação e Nutrição apresentando-se como um estudo original na literatura diante da ausência de estudos nessa população específica.

A maioria dos trabalhadores tinha idade menor ou igual a 45 anos, considerando-se uma amostra relativamente de adultos jovens (entre homens e muIheres). Os trabalhadores apresentavam frequências equivalentes em relação ao tempo de serviço, que em média era de 24 meses.

Em relação aos níveis de 25(OH)D, foi constatada uma prevalência de $61,5 \%$ de deficiência/insuficiência de VD, e mais da metade dos participantes nessa condição de hipovitaminose apresentavam sobrepeso ou obesidade, porém não foram observadas diferenças entre o status da vitamina com o estado nutricional. 


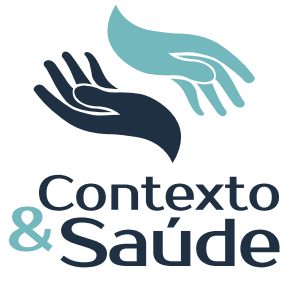

Para as variáveis que analisaram o perfil sociodemográfico e ocupacional, fototipo de pele, exposição e proteção solar, PA (sistólica e diastólica), estado nutricional e CC, não foram encontradas associações com os níveis séricos de 25(OH)D. Em relação à exposição solar, observou-se associação para "Se expõe ao sol na ida ao trabalho" com o status de 25(OH)D; descrição de associação semelhante não foi encontrada em demais estudos publicados até o presente momento. Acredita-se que pelo fato de a jornada de trabalho iniciar em horário muito cedo da manhã essa exposição solar na ida ao trabalho não teria relação com a biossíntese da VD, visto que normalmente os trabalhadores ingressam no serviço por volta das 6 horas. Na análise de regressão, essa variável mostrou-se influenciadora apenas na regressão bruta, não sendo confirmada no modelo ajustado. Ressalta-se, ainda, que o ambiente laboral desses trabalhadores caracteriza-se em condições de confinamento (estilo indoor).

Em um estudo realizado entre dois grupos ocupacionais na Coreia: um grupo de pescadores saudáveis ( $n=140$ ), mais expostos ao sol, e o grupo de trabalhadores saudáveis com ocupação geral $(n=140)$, relativamente menos expostos ao sol, verificou-se que $78 \%$ dos indivíduos do grupo de pescadores e $98 \%$ dos indivíduos do grupo de ocupação geral demonstraram deficiência (<20ng/ $\mathrm{mL}$ ) ou insuficiência (20-30ng/mL) de VD. Em relação à idade, no grupo de pescadores observou-se maiores concentrações séricas na faixa etária de 50 a 60 anos e no grupo de ocupação geral as maiores concentrações séricas de $25(\mathrm{OH})$ $\mathrm{D}$ foram encontradas nos indivíduos idosos e mais baixas na faixa dos 20 a 40 anos. $^{23}$

Em outra pesquisa, de vigilância nacional, verificou-se que indivíduos de 20 a 40 anos apresentaram níveis séricos mais baixos de 25(OH)D quando comparados com outras faixas etárias. ${ }^{24}$ Semelhantemente, no estudo realizado com enfermeiras $(n=114)$ constatou-se que a deficiência de VD foi mais notável na faixa etária de 26 a 35 anos. ${ }^{13}$ No presente estudo, entre os trabalhadores que apresentaram deficiência/insuficiência de VD foi observado que a maioria tinha idade menor ou igual a 45 anos, corroborando os estudos anteriores. Esses achados, possivelmente, devem-se ao fato de que os níveis de 25(OH)D podem sofrer a influência de uma gama de fatores como exposição solar, idade, predisposição genética e ingestão alimentar. ${ }^{23}$

Quanto aos parâmetros bioquímicos analisados não foram observadas diferenças entre as médias e o status de vitamina $D$, assim como para os marcadores inflamatórios analisados (PCR e AGP). Na análise de regressão de prevalência ajustada, no entanto, verificou-se que as variáveis bioquímicas glicemia de jejum (Diabetes Tipo 2) e triglicerídeos (muito alto) mostrou-se com associações significativas em relação ao status de VD.

Batista et al. ${ }^{25}$ relatam que trabalhadores que apresentavam hipovitaminose $D(<30 \mathrm{ng} / \mathrm{mL}$ ), tinham uma chance 5,9 vezes maior de ter o colesterol-LDL alterado e uma chance de 2,3 vezes maior de triglicerídeos alterados, se comparados aos trabalhadores com níveis suficientes. Em um estudo transversal desenvolvido em uma população do Catar concluiu-se que altos níveis de triglicerídeos estavam associados a baixos níveis séricos de 25(OH)D².

Editora Unijuí - Revista Contexto \& Saúde - ISSN 2176-7114 - v. 21, n. 44, out./dez. 2021 


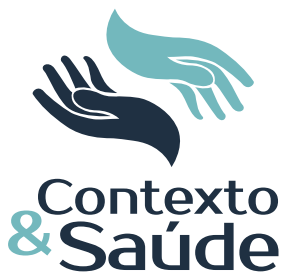

Em relação aos efeitos que a VD pode exercer no Diabetes Tipo 2 pesquisadores apontam que a deficiência de $25(\mathrm{OH}) \mathrm{D}$ altera a produção e secreção de insulina, contribuindo para o maior risco de Diabetes Tipo $2 .{ }^{2}$

Em relação ao estresse oxidativo, as concentrações médias de MDA não apresentaram diferenças entre o status de VD, enquanto os resultados de CAT apresentaram diferença nas médias entre os níveis de 25(OH)D, concluindo-se que os indivíduos que apresentam deficiência/insuficiência de VD apresentam uma menor CAT, contribuindo para o aumento do estresse oxidativo. Indivíduos com baixos níveis de VD em sua maioria apresentam níveis aumentados de marcadores inflamatórios e de estresse oxidativo. 4,6,7,26,27

As concentrações de 25(OH)D abaixo do ideal aumentam o dano oxidativo intracelular, tendo o calcitriol efeitos benéficos na regulação da atividade antioxidante. ${ }^{28} \mathrm{~A}$ interação do calcitriol, forma biologicamente ativa da VD, com seus receptores intracelulares modula a transcrição gênica dependente da VD e a ativação de elementos responsivos à VD, desencadeando múltiplos sistemas mensageiros. Assim, a VD tem ação oxidante, favorecendo o equilíbrio das atividades mitocondriais, evitando a oxidação de proteínas relacionadas ao estresse oxidativo, peroxidação lipídica e danos ao DNA., 428

Nesta pesquisa foi proposta a investigação da prevalência de hipovitaminose $\mathrm{D}$ em trabalhadores e as possíveis associações dessa condição a parâmetros metabólicos, pois embora existam estudos sobre VD, a maioria é relacionada a doenças e poucos em população adulta saudável e grupos ocupacionais. Apresenta-se como limitação importante desse estudo, no entanto, o tamanho da amostra diante do número muito superior de trabalhadores desse segmento do setor de alimentação coletiva e o fato de não ter sido avaliada a ingestão alimentar de VD.

\section{CONCLUSÃO}

Os resultados deste estudo constataram uma alta prevalência de hipovitaminose $\mathrm{D}$ e a associação dessa condição com parâmetros metabólicos, como a glicemia e os triglicerídeos. Pode-se verificar que os trabalhadores com baixos níveis séricos de 25(OH)D apresentam menor CAT e por conseguinte maior exposição ao estresse oxidativo. A alta prevalência de deficiência de VD na população estudada suscitou a necessidade de desenvolver pesquisas adicionais sobre a temática vigilância em saúde do trabalhador. Esse achado enfatiza uma forte recomendação em relação ao monitoramento dos níveis séricos de 25(OH)D e dos parâmetros metabólicos nas políticas de saúde do trabalhador, tendo em vista as implicações importantes sobre a etiologia de várias doenças crônicas não transmissíveis.

\section{REFERÊNCIAS}

${ }^{1}$ Al-Dabhani K, Tsilidis KK, Murphy N et al. Prevalence of vitamin D deficiency and association with metabolic syndrome in a Qatari population. Nutr Diabetes. 2017;7:e263.

Editora Unijuí - Revista Contexto \& Saúde - ISSN 2176-7114 - v. 21, n. 44, out./dez. 2021 
${ }^{2}$ Rafaelli RA, Nomura PR, Figueira FD et al. Influência da vitamina D nas doenças endocrinometabólicas. Semina Ciênc Biológicas e Saúde. 2015;36:333-348.

${ }^{3}$ Ganmaa D, Holick MF, Rich-Edwards JW et al. Vitamin D deficiency in reproductive age Mongolian women: a cross sectional study. J Steroid Biochem Mol Biol. 2014;139:1-6.

${ }^{4}$ Percegoni N, Castro JM de A. Vitamina D, sobrepeso e obesidade - uma revisão. HU Rev. 2014;40. [Acesso em: 13 dez. 2019]. Disponível em: https://periodicos.ufff.br/index. php/hurevista/article/view/2456

${ }^{5}$ Palacios C, Gonzalez L. Is vitamin D deficiency a major global public health problem? J Steroid Biochem Mol Biol. 2014;144 Pt A:138-145.

${ }^{6}$ Jorge AJL, Cordeiro JR, Rosa MLG et al. Vitamin D Deficiency and Cardiovascular Diseases. Int J Cardiovasc Sci. 2018;31:422-432.

${ }^{7}$ Hossein-nezhad A, Holick MF. Vitamin D for health: a global perspective. Mayo Clin Proc. 2013;88:720-755.

${ }^{8}$ Maeda SS, Borba VZC, Camargo MBR, et al. Recomendações da Sociedade Brasileira de Endocrinologia e Metabologia (SBEM) para o diagnóstico e tratamento da hipovitaminose D. Arq Bras Endocrinol Amp Metabol. 2014;58:411-433.

${ }^{9} \mathrm{Gu}$ JK, Charles LE, Millen AE, et al. Associations between adiposity measures and 25-hydroxyvitamin D among police officers. Am J Hum Biol Off J Hum Biol Counc. 2019;31:e23274.

${ }^{10}$ Cheng Q, Du Y, Hong W, et al. Factors associated to serum 25-hydroxyvitamin D levels among older adult populations in urban and suburban communities in Shanghai, China. BMC Geriatr. 2017;17. Epub ahead of print 24 out. 2017. doi: 10.1186/s12877-0170632-z.

${ }^{11}$ Unger MD, Cuppari L, Titan SM et al. Vitamin D status in a sunny country: where has the sun gone? Clin Nutr Edinb Scotl. 2010;29:784-788.

${ }^{12}$ Fajardo VC, de Oliveira FLP, Machado-Coelho GLL et al. Effects of vitamin D supplementation on cardiovascular risk factors in shift workers: Study protocol for randomized, double-blind, placebo-controlled clinical trial. Medicine (Baltimore) 2019;98:e15417.

${ }^{13}$ Hamid R, Ahmad K, Golshan F et al. Vitamin D Deficiency among Female Nurses of Children's Medical Center Hospital and Its Related Factors. Acta Medica Iranica. Acta Medica Iranica, 2016;54:146-150.

14 Brasil. Resolução CNS no. 466, de 12 de dezembro de 2012 - Estabelece as diretrizes e normas brasileiras regulamentadoras de pesquisas envolvendo seres humanos. Rev Bras Bioét. 2012;8:105-120.

${ }^{15}$ Sociedade Brasileira de Dermatologia. Classificação dos fototipos de pele. [Acesso em: 13 dez. 2019]. Disponível em: http://www.sbd.org.br/dermatologia/pele/cuidados/ classificacao-dos-fototipos-de-pele/

16 WHO. Obesity: preventing and managing the global epidemic. [Acesso em: 22 jul. 2016]. Disponível em: http://www.who.int/entity/nutrition/publications/obesity/ WHO_TRS_894/en/index.html

${ }^{17}$ Sociedade Brasileira de Cardiologia AM. 7a Diretriz Brasileira de Hipertensão Arterial. 2016;107:1-83.

${ }^{18}$ Nargesi AA, Ghazizadeh Z, Larry M et al. Manual or automated sphygmomanometer? A historical cohort to quantify measurement bias in blood pressure recording. J Clin Hypertens Greenwich Conn. 2014;16:716-721.

${ }^{19}$ Holick MF, Binkley NC, Bischoff-Ferrari HA et al. Evaluation, treatment, and prevention of vitamin D deficiency: an Endocrine Society clinical practice guideline. J Clin Endocrinol Metab. 2011;96:1911-1930.

${ }^{20}$ Duarte PS, Decker HH, Aldighieri FC et al. Relação entre os níveis séricos de cálcio e paratormônio e a positividade da cintilografia das paratiróides com sestamibi: análise de 194 pacientes. Arq Bras Endocrinol Amp Metabol, 2005;49:930-937.

${ }^{21}$ FERRACI CKBF. Total antioxidant capacity (CAT) in clinical, experimental and nutritional studies. Journal of the Health Sciences Institute. 2010;28:307-310. 
${ }^{22}$ Antunes MV, Lazzaretti C, Gamaro GD et al. Estudo pré-analítico e de validação para determinação de malondialdeído em plasma humano por cromatografia líquida de alta eficiência, após derivatização com 2,4-dinitrofenilhidrazina. Rev Bras Ciênc Farm. 2008;44:279-287.

${ }^{23}$ Lee D-H, Park KS, Cho M-C. Laboratory confirmation of the effect of occupational sun exposure on serum 25-hydroxyvitamin D concentration. Medicine, Baltimore. 2018;97:e11419.

${ }^{24}$ Choi HS, Oh HJ, Choi H et al. Vitamin D insufficiency in Korea--a greater threat to younger generation: the Korea National Health and Nutrition Examination Survey (KNHANES) 2008. J Clin Endocrinol Metab. 2011;96:643-651.

${ }^{25}$ Batista AP, Ambrosim TV, Nascimento Neto RM do et al. Hypovitaminosis D is associated with visceral adiposity, high levels of low-density lipoprotein and triglycerides in alternating shift workers. [Acesso em: 13 dez. 2019]. Disponível em: http://www.repositorio.ufop.br/handle/123456789/8774

${ }^{26}$ Holick MF. Vitamin D status: measurement, interpretation, and clinical application. Ann Epidemiol. 2009;19:73-78.

${ }^{27}$ Kratz DB, Silva GS e, Tenfen A. Deficiência de vitamina D (250H) e seu impacto na qualidade de vida: uma revisão de literatura. Rev Bras Anal Clin. 2018;118-123.

${ }^{28}$ Wimalawansa SJ. Vitamin D Deficiency: Effects on Oxidative Stress, Epigenetics, Gene Regulation, and Aging. Biology; 8. Epub ahead of print 11 May 2019. doi: 10.3390/ biology 8020030 